\title{
Streptococcus salivarius Group
}

National Cancer Institute

\section{Source}

National Cancer Institute. Streptococcus salivarius Group. NCI Thesaurus. Code C86805.

A bacterium that belongs to the Salivarius group of the genus Streptococcus. 